\title{
Social Fringe Dwellers: Can chat-bots combat bullies to improve participation for children with autism?
}

David Ireland

\section{Geremy Farr-Wharton}

DanaKai Bradford

\author{
Australian e-Health Research Centre \\ D.Ireland@csiro.au \\ Corresponding Author
}

Australian e-Health Research Centre

Geremy.Farr-Wharton@csiro.au

Australian e-Health Research Centre

Dana.Bradford@csiro.au

Autism Spectrum Disorder (ASD) can cause a gulf in communication that casts children with autism to the fringes of social and family life, despite the best efforts of their carers. These children often struggle with social interaction, lack of interest and empathy, and require intensive therapy to improve their ability to communicate with others. Improvements in social interaction are often hampered by experiences in which children with autism are more susceptible to being bullied. Social and communication technologies (e.g. smartphones and tablets), which children with autism tend to gravitate toward, and to which many families have access, may play a significant future role in building resilience and improving social interaction. Based on technology reviews and stakeholder interviews, we are developing modules for a machine learning artificial intelligence platform (a chat-bot) that assists children attending an Australian mainstream school to recognise and respond to social bullying and sarcasm, allowing bullied autistic children to develop the social prowess to withstand their aggressors.

Keywords: Autism Spectrum Disorder, bullying, digital technologies

Ireland, D., Farr-Wharton. G., Bradford, D.K. (2018). Social fringe dwellers: can chat-bots combat bullies to improve participation for children with autism? The Journal of Community Informatics, 14(1), 105--119.

Date submitted: 2018-10-01. Date accepted: 2018-10-15.

Copyright (C), 2018 (the author as stated). Licensed under the Creative Commons AttributionNonCommercial-ShareAlike 2.5. Available at: www.ci-journal.net/index.php/ciej/article/view/1454 


\section{Introduction}

The digital fringe refers to people who are generally excluded in the design of digital solutions due to lack of access, lack of resources or social exclusion (Davis et al., 2017). Yet there are some instances where social exclusion fosters digital inclusion. In these cases, practical, innovative and sensitive design solutions are required to draw these people in from the social fringes. This article is based on the premises that the nature of autism spectrum disorder (ASD) can lead to social exclusion for children even within mainstream communities and that these children tend to gravitate toward technologies to facilitate learning. Children with autism are more susceptible to being bullied, further compounding social isolation. We therefore propose that utilising this tendency for digital participation, in this marginalised population, may provide a solution for children with autism to recognise and respond to social bullying.

People who experience bullying report low self-esteem, mental illness, propensity to self-harm and suicide ideation, which may persist well after the bullying has ceased (Hebron \& Humphrey, 2014). Risk factors that increase the probability of being bullied include ethnicity, poor social interaction, and low academic success (Lodge, 2014). Conversely, social networks have been found to offer a significant protective factor in minimising the extent of bullying (Hebron \& Humphrey, 2014), and consequently, the ongoing effects.

Special needs children, particularly those with autism, have been identified as being more likely to be bullied (Hebron \& Humphrey, 2014; Anderson, 2014), potentially partly due to the inherent difficulties they face in forming positive relationships in social networks. Sadly, Anderson (2014) found that autistic children who have a desire to interact with other children were bullied more frequently than children who preferred to play alone. The longevity of the effects of bullying and the impact of those effects on an already vulnerable population indicate the need for sensitive long-term solutions that are easily integrated into everyday lifestyles.

\section{Living on the fringe}

ASD is a complex lifelong condition that becomes apparent from early childhood. The spectrum denotes an array of neurodevelopmental disorders with associated impacts on functioning (ranging from mild to severe). While each individual is different, many struggle with communication, social interaction, and sensory processing, and may exhibit deficient or repetitive behaviour and a lack of interest and empathy (Patel, Preedy \& Martin, 2014). In Australia, 1 in every 100 individuals (approximately 230,000) have ASD and together they comprise the second largest representative group $(29 \%)$ of those who receive funding from the National Disability Insurance Scheme (NDIS) (NDIS 2018, p. 33).

There is strong evidence to suggest that early diagnosis of ASD can lead to better outcomes for the individual later in life (Christensen et al., 2016). However, while ASD 
assessments include a complex array of behavioural, psychosocial, and movement kinematics monitoring, the age that diagnosis occurs can vary significantly, with most between the ages of 2 and 8 years old. Early diagnosis tends to occur more often for males than females, because symptom manifestation can be more prominent and perceptible in males (Kreiser \& White, 2014). For females, the subtlety of symptom manifestation can result in no diagnosis, misdiagnosis, or a significantly delayed diagnosis. The earlier accurate diagnosis occurs, the greater the impact of interventions and therapy in promoting higher function in later years.

Interventions are generally implemented at various stages of childhood development, and are tailored towards the severity of the neurological condition. Individuals with autism are more likely to have other neurological conditions, such as an intellectual disability, which can impact upon the treatment options available. As a result, professionals and practitioners will generally select appropriate interventions that are evidence-based and have demonstrated positive impact for individuals with ASD (Wong et al., 2015). Reviews of the evidence-based practices commonly used to assist individuals with ASD at various ages and stages of development are readily available (Wong et al., 2015; Ospina et al., 2008). Most interventions are aimed at impacting and improving communication and social interaction, behaviour and play, academic prowess, cognitive function and ability, employability, movement and motor skills, and mental health and emotional wellbeing.

Learning deficiencies are common among individuals with ASD, although severity can vary significantly. Such deficiencies can make it difficult for children with ASD to develop new and diverse strategies for working through and dealing with interactions with others in complex situations. Learning deficits hamper the ability of the individual to engage in social and cultural understanding, potentially exacerbating other deficiencies associated with their autism condition (Jordan, 2013), such as learning subtle social practices and emotions through interactions with others. Individuals can become overwhelmed, disconnecting and withdrawing from life-events, scenarios or interactions for which they may have low tolerances and that negatively impact them (e.g. low tolerance to sensory stimuli). A subgroup of children with autism are 'visual learners', requiring information to be presented in a way that can be seen. These children often have difficulty analysing the meaning of oral instruction (Tissot \& Evans, 2010). Early intervention, therefore, aims to facilitate learning incorporating the unique complexities associated with autism in a given individual (Jordan, 2013).

Early difficulties with social interaction lead to challenges in maintaining both personal and professional relationships. As a result, individuals with autism often struggle with employment throughout their adult life, reflected in an unemployment rate of more than 50\% in both Australia (Scott et al., 2017, p. 2) and America (Wehman et al., 2014, p. 1). In the UK, only $15 \%$ of people with autism have full time paying jobs, and approximately one-third of those who have joined the workforce in any capacity have experienced bullying in the workplace (Bancroft, Batten, Lambert \& Madders, 2012, p. $15)$. 


\section{The bully's advantage}

Identifying bullying in children with ASD is hampered by the fact that children with ASD may have limited speech and struggle to communicate their experiences. In these cases, being bullied can compound social interaction difficulties and impede communication development. Moreover, some individuals struggle with understanding social interactions and may not know themselves whether they are being bullied. This is particularly common with indirect bullying such as social exclusion (Hebron \& Humphrey, 2014) and makes it all the more difficult to support students being bullied. An Australian-wide study found that $80 \%$ of adults with autism reflected that they had not received sufficient support for bullying experienced during their education (ASPECT, 2012, p. 27). Many did not complete their education because of bullying, and thus have difficulty supporting themselves financially, as evidenced by the high unemployment rates cited above. The prevalence of bullying beyond the schoolyard, and into social media and the workforce, together with its strength as a predictor for reduced quality of life in able adults with autism (Hong, Bishop-Fitzpatrick, Smith, Greenberg \& Mailick, 2016), strongly supports early implementation of programs to build lifelong resilience against bullying behaviour.

Once a child has been identified as either likely to be or being bullied, it is important that they are taught strategies and skills on how to recognise and respond to the social situation. Guidelines for developing anti-bully plans are becoming commonplace in most schools; however, innovative methods are required for teaching children with autism as they may have difficulty perceiving the concept of bullying and social betrayal. Methods of teaching involving technology are becoming more appealing for children with autism as these individuals tend to gravitate towards technology (Boser et al., 2014). It is believed that the visual focus and having the locus of control account for some of the appeal (Ennis-Cole, 2015). Social skills programs, such as the Secret Agent Society, that incorporate technology including role play and computer games, have been shown to be effective in improving the ability of people with autism to recognise emotions and manage bullies (Beaumont, Rotolone \& Sofronoff, 2015).

At this point in time, it is not known which form of technology is best able to help an autistic individual understand and respond to bullying behaviour. We conducted app reviews and stakeholder interviews to help determine how technology could benefit children with autism in general.

This article contributes to the development of a strategy for designing digital technologies for children on the autism spectrum. In particular, we explore a technology in the form of an artificial intelligence (AI) conversation agent (commonly referred to as a chat-bot). This technology could play a unique role as a digital intervention in the management and building of resilience to bullying behaviour. We summarise our recent study, which examined how families and children on the autism spectrum are accessing and using mobile technology. We then present our AI chat-bot, HARLIE, which we are developing for individuals with neuro-developmental impediments, including autistic children, and the lessons from previous work on which we are basing HARLIE's future design. Finally, we discuss the implications of our findings, and the future role of 
technology in improving outcomes for individuals on the spectrum and conclude with a summary of the next steps associated with our work.

\section{Designing participatory technologies}

Insight into the development of technologies for people with autism can be gleaned from successes and failures of previous technologies. To determine what apps were already available and what aspects of those apps were beneficial for children with autism, we used a web crawler to examine 56 million reviews from more than two million apps. The full mobile app review is detailed elsewhere (Ireland, Bradford \& Silvera-Tawil, 2017).

\section{Mobile App Review Summary}

In summary, of the 56 million app reviews downloaded from the Apple App and Google Play Stores, about 1 in 7,500 reviews from Apple and 1 in 50,000 Android reviews contained useful information. Only 57 apps were found that were specifically designed for people with autism and claimed to be evidence-based. More than $85 \%$ of the reviews were for an app that was neither designed nor advertised for people with autism. This was confirmed by manually reading the description of each app for mention of its intended users. Despite the small number of ASD specific apps, children with autism were reported to have experienced benefits, particularly in language, education and attention. Reviews with poor ratings usually reported cost, followed by functional issues and advertisements. For our purposes in designing participatory technologies, the functional issues are of most import. The consequences of a malfunctioning mobile app were clearly illustrated by a parent:

Bought this app for my 5 yr old with autism. He loves Disney. App always crashes so now all he does is scream in frustration when it repeatedly doesn't work.

After technical issues, the greatest source of frustration and distress came from developers making changes to the app appearance by adding or removing features, changing interfaces or upgrading colour schemes. A specific example of this occurring was detailed by one parent with a child on the autism spectrum whose favourite game was changed by the developers:

This was my son's favourite game. My son is autistic. A seemingly small change like this is life-altering drama for him. Please change the icon, at least, so he thinks it's a different game.

Had these apps been developed with, for and by people in the autism community, using a participatory/co-design approach, developers could have taken the necessary steps to avoid causing distress (Ireland, Bradford \& Silvera-Tawil, 2017). For our bullying module, this means that beta versions are unadvisable, and that all developed and tested scenarios need to remain accessible. 


\section{Expert interviews in the wider autism community}

As part of our initial research design, we implemented expert interviews (as described by Flick, 2014) with seven individuals in the wider autism community, targeting their understanding of the crucial considerations when developing technologies to support children with ASD (Farr-Wharton, Ireland, Good, Chang \& Bradford, 2017). Expert interviews were uniquely beneficial to this task, in comparison with other interview techniques, because of the complexities and sensitivity associated with autism. For ethical reasons, this limited the scope of interviews to key stakeholders involved with children on the spectrum. The stakeholders we engaged for interview included a parent of children with autism (male, referred to as P1 when quoted), two teachers who work with children with autism (males, quoted as T1, T2), two speech therapists who have worked extensively with children with autism (females, quoted as ST1, ST2), and two occupational therapists with significant experience working with children with autism (females, quoted as OT1, OT2).

The questions asked of participants centred on three specific topics: (a) challenges most commonly experienced with the children, (b) key barriers perceived to make monitoring progress of a child challenging for parents, therapists and teachers, and (c) solutions already explored to overcome some of the challenges faced in their role.

\section{Expert interview insights}

The responses from the expert interviews provided emergent themes regarding the challenges participants have faced in their roles when interacting with children with ASD and the potential opportunities for overcoming the perceived barriers. There were two underlying themes that emerged strongly from their responses: (a) There are distinct challenges in monitoring the development and progression of children with autism and communicating this effectively with the 'care team' (i.e. family, carers, clinicians and teachers), (b) Parents and carers are continuously seeking innovative tools that enable improved social inclusion and encourage quality family-time with their child(ren).

\section{Challenges for Monitoring Development and Progression}

Our clinician and teacher participants (i.e. ST1, ST2, OT1, OT2, T1, and T2) explained that monitoring development is challenging, particularly outside of a clinical or classroom setting, where children may become disengaged with the content in focus. Clinicians may miss key opportunities to examine new skills and progress, particularly when these skills may have been learned and implemented in the home, away from observation. In these instances, clinicians often rely on parents to record (through video), the occurrences of when behavioural or interaction development is being demonstrated or exhibited by the child. This is because clinicians need to physically observe changes within skills to adequately assess development progression and tailor the therapy they provide. For example, ST1 and OT1 both suggested that parents can be enthusiastic about their child's developmental progression and may be prone to embellish instances when they noticed a child improving their skills, which have previously been identified as deficient. P1 also stated that he can sometimes find it 
difficult to explain progress to a clinician, as they will often ask for the behaviour (or skill) to be repeated in the clinical setting. In some instances, the child can become disengaged from the clinical setting and will not necessarily repeat the behaviour. In these instances, the parent is encouraged to record the behaviour when it is enacted at a later time for demonstration at their next consultation with the occupational therapist. As there are challenges and complexities associated with this process, occupational therapists and speech therapists are seeking new tools that enable better capture of behavioural and skill development in children.

Teachers experience similar challenges with monitoring the progress of children with ASD. T1 and T2 both spoke to the challenges faced by teachers to provide a tailored learning experience to a child, particularly when non-ASD children also require learning assistance in the same classroom. This problem is exacerbated as the classroom size increases. T1 also stated that there were challenges in facilitating parents' expectations of providing tailored learning to suit their child's needs, as there are limits for how to achieve this cohesively. T1 suggested that to achieve this cohesion, there needs to be a common message, instruction, and strategy that is implemented regardless of location (e.g. home or in the classroom). This requires the ability for parents and teachers to communicate more effectively and often to ensure synergy is achieved in messaging. T2 also provided the perspective that a monitoring and communication platform would break down barriers for children with ASD and improve the learning they ultimately receive.

\section{‘Tools' for Improving Social Inclusion and Quality Family-time}

Many of our participants (e.g. OT1, ST1, OT2), and in particular P1, mentioned the need for targeted tools for parents and carers, which are tailored to their child's context, and encourage quality family-time and inclusion. P1 suggested that enabling quality family-time and inclusion would help their child to continuously improve the development of their skills within the home setting. P1 also suggested that they continually seek new apps that positively engage and stimulate their child in developmental opportunities, rather than those that promote repetitive, addictive or negative engagement (e.g. 'watching the same Youtube video over and over' (P1)). Specifically, P1 mentioned that the technology they seek needs to be dynamic in how it responds to the child, and 'common scripts' (P1) or features must be implemented so that the child is able to utilise 'standard' interactions if they are unsure of what do to next. OT $(1,2)$ and $\operatorname{ST}(1,2)$ also spoke to the opportunities for technology to engage ASD children at home, where that technology would assist in leading to positive outcomes for the child, such as cultivating social interactions skills.

P1 spoke to the challenges associated with purchasing expensive therapeutic technology for their child. Often, parents will try to support the development of their ASD child, as best as they can, through improving their access to therapeutic goods and services, particularly those that are easy and accessible (e.g. apps for their phone or tablet). However, P1 mentioned that he, and others in a similar situation, have limited resources to rely on within the home, as their government-funded assistance is finite and is usually allocated to recommended services (e.g. occupational/speech therapists and 
intervention programs). This leaves parents with little resources to spend on other tools and supports, often requiring personal investment in such interventions, which may not have any scientific evidence to support their claims, reducing the incentive to invest in 'higher risk' opportunities (e.g. services that have little-to-no evaluation of their effectiveness).

In some instances, children with ASD may also require extended time with their parents and carers, which can be demanding. P1, OT1 and ST1 spoke to the need for tools that can support parents and carers in providing interaction time with their children. In these instances, P1 suggested that if a tool can supplement a part of the extended time their child required for interaction, while still providing the positive outcomes for the child, this would help them as a parent immensely. They would be able to reallocate this time to recuperation and for other necessary activities. However, producing opportunities for co-interaction between parents/carers and children with ASD, which is facilitated or aided by a stimulus, may encourage opportunities for quality family time to develop.

On the basis of our analysis of app reviews and stakeholder interviews, we determined that a chat-bot would be an ideal technology to deliver information and resources around bullying behaviour to children with autism. The chat-bot meets the need identified by teachers, parents and clinicians for monitoring development of children with ASD while providing the benefits of language development identified through our app review analysis.

\section{The rise of chat-bot technologies}

With the advent of social and communication technologies, conversation agents, or chat-bots, provide a means for users to become engaged in conversation, continuing and progressing the dialogue in the same way human-to-human interaction occurs. They have more recently been used within the health paradigm for their ability, amongst other benefits, to autonomously gather key data about the user for analysis by clinicians. Ultimately, this data can be used to tailor and improve the care clinicians provide. Some examples where chat-bots have been implemented previously, include: (a) encouraging physical activity in overweight adults (Watson, Bickmore, Cange, Kulshreshtha \& Kvedar, 2012), (b) encouraging behaviour change (Bickmore, Gruber \& Picard, 2005), and (c) providing information relating to sex, drugs and alcohol (Crutzen, Peters, Portugal, Fisser \& Grolleman, 2011).

Chat-bots present a useful tool to support the development of language and communications skills in users. Chat-bots also provide stress-free interaction without social anxiety, which allows users to practice and refine their communication skills unencumbered. Tailoring the level of difficulty to suit the user's growth can further nurture their skills. With these capabilities, users can practice and develop their social interaction skills with the chat-bot, prior to engaging with others face-to-face. This process will empower them to feel more confident during social interaction with others.

Families with children who have been diagnosed with ASD receive government-funded support, providing subsidised access to clinicians, therapists and service providers. 
These services are often provided outside of the home, and for some children, this can add to anxiety, stress and disengagement, reducing their effectiveness. Access to funding decreases after the age of seven and families rely more heavily on their own resources to continue supporting their child's development. Families may often seek out new support tools and mechanisms that will help their child continue their developmental journey throughout childhood and adolescence.

Children with ASD can find social interaction challenging and frustrating, which can lead to social disengagement and result in social isolation. Improving their social interaction skills is essential. To do this, parents, speech pathologists and occupational therapists are heavily relied on to identify strategies that will support the child's progression. Parents can also gain assistance through service providers and charities. However, aside from the time parents can spend with their child, there is little assistance children can receive to improve their social interaction skills outside of the clinical setting. Chat-bots present a potential solution to this problem. They can be used to engage with a child outside and within the home, becoming a comfortable personal assistant that can nurture the social interaction skills of a child.

\section{Introducing HARLIE}

HARLIE (Human and Robot Language Interaction Experiment) is a chat-bot we are developing that is akin to Apple's Siri (Ireland et al., 2016; Ireland, Bradford \& FarrWharton, 2017). Various modules allow conversations on a variety of topics such as interests and hobbies, weather, identifying sarcasm, as well as condition specific modules such as speech pathology, neurodegeneration and clinical care.

HARLIE is very extensive and leverages state-of-the art technology in speech detection and natural language processing. None-the-less, expertise in computer science is not needed to develop content for HARLIE's 'brain'. In fact, a majority of the existing content was developed by speech and occupational therapists. HARLIE allows for multi-modal interaction in the form of voice, text and images. For non-verbal children, an interface using the picture exchange communication system (National Autism Resources, 2017) is also being developed.

For individuals with developing speech, HARLIE can analyse the user's voice and communication. This includes measuring speech and syllable rate; how vowels are articulated; vocabulary range and duration of mid-sentence pauses (Figure 1). These metrics could be helpful for parents and clinicians to measure speech improvement in children with autism as well as for researchers who are trying to understand the challenges and benefits for the individual. Log data analysis in longitudinal studies could determine the impact of social interaction on communication development.

\section{Building a bullying module}

HARLIE's bullying module is under development pending co-design by the autism community. A number of key stakeholders have expressed interest in being involved in the evolution of this module for children, young adults in tertiary education and young 
employed adults with autism. Early content includes an example interaction of the beginning of a conversation involving identifying whether the user is being bullied (Figure 2). Images are embedded into the responses to help guide the meaning of the conversation.

Content for the bullying module is being collected from the wider autism community through several mechanisms including interviews, focus groups, workshops and most excitingly, via a portal through which people with autism can log into and upload content directly to the 'brain' of the bullying module. All content will be moderated before being made available through the chat-bot.

Various scenarios are being designed for the chat-bot that allow the user to work through different responses and reactions. These are currently being developed in conjunction with children and adults with autism, parents, educators and clinicians.

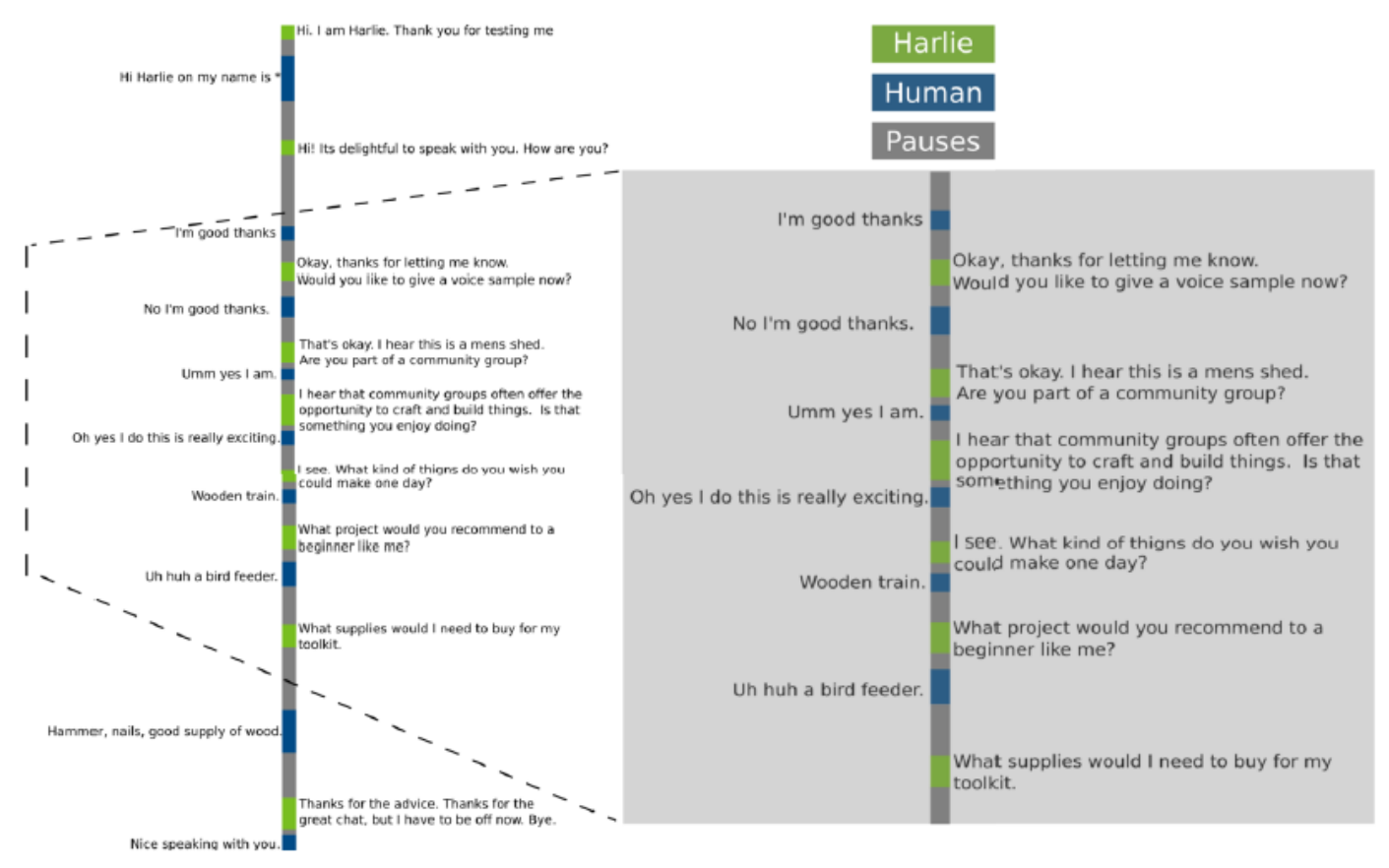

Figure 1: A sample dialogue between HARLIE and a person with autism. Green rectangles designate dialog generated from HARLIE, blue rectangles designate responses from the user, grey rectangles indicate pauses in the timeline (Ireland et al., 2016). 


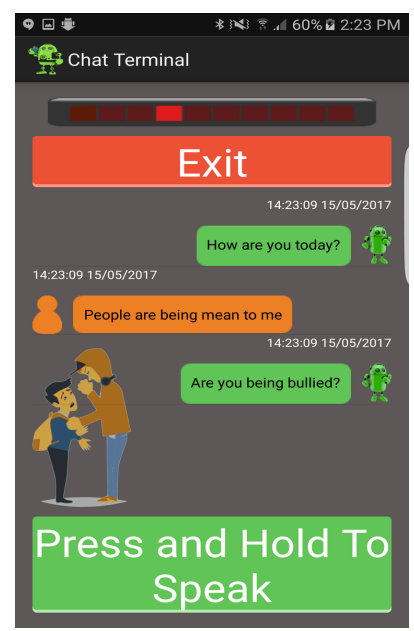

Figure 2: An example of a conversation to identify if a child perceives they are being bullied.

\section{Discussion}

HARLIE, a chat-bot AI app, was originally designed for a clinical speech pathology population. Over the course of its development, and commensurate with an autism focused review of existing apps together with stakeholder interviews, it became clear that HARLIE was the ideal technology to deliver a bullying module to help build resilience in children with autism.

Our stakeholder interviews revealed that parents, clinicians and teachers are interested in technology to support children with Autism. Specifically, they are interested in technology that reflects development, dynamically responds to the child, allows for cointeraction with family to facilitate quality time, and is affordable. Clear guidelines for software development emerged from our analysis of existing app reviews. Based on this preliminary research, we are currently conducting further developments to the bullying 'brain' with, by, and for the wider autism community. In the first instance, the bullying module is being developed to complement school bullying programs.

\section{The technologies targeting bullying in school}

Within schools, a variety of technologies have been identified for their efficient delivery of bullying-related information and for the interest they inspire within the younger generations. For this reason, integrating interventions with technologies may be one of the most effective means of engaging younger generations and improving the effectiveness of interventions. For example, virtual learning has emerged as a key tool used in schools to build resilience in victimised children and is having a positive impact in reducing victimisation in general (Sapouna et al., 2010).

The roles of technology-leveraged interventions targeting bullying in schools have differed significantly depending on their focus of intervention (e.g. a focus on bullying or victimisation, age groups, and gender). However, many interventions have not been formally evaluated for their efficacy in minimising bullying or victimisation (Sapouna 
et al., 2010). This is of growing importance as new forms of bullying emerge with the new technologies becoming available to the consumer market (e.g. cyberbullying). With the emergence of cyberbullying, the 'boundaries' of when and where bullying can take place have swelled, no longer centred to the home or school environment. Even the predators are increasingly anonymous to the victim ( $\mathrm{Li}, 2007)$. The prevalence and impact of bullying has also increased, which will continue to have implications for the roles that interventions will need to play in preventing or mitigating opportunities for bullying, or reducing victimisation and its impacts through building resilience in victims, enabling them to navigate complex and challenging social situations.

Ttofi and Farrington (2011) stress the importance of the role of schools in providing bullying and victimisation information, as well as interventions to reduce both. Their study identified that common interventions implemented within school are reducing bullying and victimisation by between $20-25 \%$, which also suggests there is room for improvement.

To reduce marginalisation, it is important that bullying programs designed for and by people with autism complement those implemented in mainstream schools.

\section{A final note on designing participatory technologies}

Designing a device so that it has the desired impact, requires considerable expertise from technology developers and members of the wider autism community. This work sought insight from this latter group by summarising the results of a web-crawler that scanned reviews of mobile apps that were related to autism spectrum and captured the essential data on how the apps were of benefit and in some cases, how harm was caused by app developers modifying the app. Unbeknownst to the developer, autistic users often find change confronting and distressing. Despite the large amount of research being done on technology uses in autism, only a relatively small number of apps were found to be designed specifically for this cohort. We found no indication that any of the apps had been developed by this cohort. To gain greater insights into this group, members of the wider autism community were interviewed regarding remote monitoring and technology that is customisable to the user, with several themes emerging. Future work on incorporating the bullying module in the HARLIE chat-bot and its eventual efficacy test will endeavour to consider these issues.

While chat-bots are arguably seen as the sexy new solution to autism intervention, continuous improvements must be made to existing interventions and all emerging interventions need to be validated for their efficacy to drive real-world outcomes for individuals on the spectrum.

\section{Conclusion}

Children with autism are more susceptible to bullies due to their neurodiversity, difficulties in initiating and understanding social interaction, repetitive behaviours and heightened sensitivity to environmental stimuli, all of which make them vulnerable to aggravation. They can be innocent of the strategies underlying the power differential 
that is characteristic of the bully-victim relationship, and may not perceive that they are being manipulated or ridiculed.

To develop technologies to support children with autism to identify and appropriately respond to bullying, we first needed to understand the benefits of technology, and the features that were not beneficial, in the eyes of children with autism and their families. Our analysis of reviews of existing apps found that apps had the potential to improve social interaction by enhancing language, education and attention skills. Normal software development trajectories, which tend to involve producing a beta version and making improvements in response to user feedback, were inappropriate for this user population. Our stakeholder interviews suggested a need for an affordable monitoring technology, built to dynamically respond to the child. On the basis of these preliminary studies, we propose the concept of using a conversational agent (chat-bot) to provide a pseudo conversation partner for practicing various oral and visual scenarios to build resilience against bullying.

The life-long difficulties a person living on the autism spectrum faces are well known and have, to a relevant extent, been described in this article. A major element of these difficulties is in handling complex social interactions and forming positive and fruitful relationships with other people. Social isolation, vulnerability to bullying behaviour and poor mental health are some of the consequences associated with ASD. Digital participation may provide the answer for social marginalisation experienced by children with ASD, especially when it comes to bullying. A chat-bot designed to help autistic children recognise and react appropriately to aggressive behaviour in their peers may assist in developing the social prowess required to develop resilience against bullies.

\section{Acknowledgements}

The authors would like to thank Anabelle Cooper, Norm Good and Emily Chang for their contribution to this work. 


\section{References}

Anderson, C. (2014). IAN Research Report: Bullying and Children with Autism Spectrum Disorder, Interactive Autism Network.

ASPECT (2012). We Belong. Retrieved from Frenchs Forest, Sydney: http:// www.autismspectrum.org.au/content/we-belong-key-findings.

Bancroft, K., Batten, A., Lambert, S., \& Madders, T. (2012) The way we are: autism in 2012. National Autistic Society, UK.

Beaumont, R., Rotolone, C., \& Sofronoff, K. (2015). The Secret Agent Society Social Skills program for children with high-functioning autism spectrum disorders: A comparison of two school variants. Psychology in the Schools, 52(4), 390-402.

Bickmore, T., Gruber, A., \& Picard, R. (2005). Establishing the computer-patient working alliance in automated health behavior change interventions. Patient Education and Counseling, 59(1), 21-30.

Boser, K., Lathan, C., Safos, C., Shewbridge, R., Samango-Sprouse, C., \& Michalowski, M. (2014). Using theraputic robots to teach students with autism in the classroom. In K. Boser, M. Goodwin, \& S. Wayland (Eds.) Technology Tools for Students with Autism (pp. 85-104). Baltimore, MD: Paul H. Brooks Publishing Co.

Christensen, D. L., Bilder, D. A., Zahorodny, W., Pettygrove, S., Durkin, M. S., Fitzgerald, R. T., Yeargin-Allsopp, M. (2016). Prevalence and characteristics of autism spectrum disorder among 4-year-old children in the autism and developmental disabilities monitoring network. Journal of Developmental and Behavioral Pediatrics, 37(1), 1-8.

Crutzen, R., Peters, G.-J. Y., Portugal, S. D., Fisser, E. M., \& Grolleman, J. J. (2011). An artificially intelligent chat agent that answers adolescents' questions related to sex, drugs, and alcohol: an exploratory study. Journal of Adolescent Health, 48(5), 514-519.

Davis, H., Hespanhol L., Farmer J., Fredericks J., Caldwell G., and Hoggenmueller M. (2017). Designing Participation for the Digital Fringe. In Proceedings of the 8th International Conference on Communities and Technologies (pp. 321-324). New York: ACM.

Ennis-Cole, D. (2015) Technology for Learners with Autism Spectrum Disorders, Switzerland: Springer International Publishing.

Farr-Wharton, G., Ireland, D., Good, N., Chang, E., and Bradford, D. (2017). Social Fringe Dwellers: Designing Chat-bots as Bridges for Children with ASD. Digital Outreach: Designing Technologies for Diversity, Participation and Social Inclusion, an OZCHI workshop. Brisbane, Australia.

Flick, U. (2014). An Introduction to Qualitative Research. London: Sage.

Hebron, J. \& Humphrey, N. (2014). Exposure to bullying among students with autism spectrum conditions: A multi-informant analysis of risk and protective factors. Autism 18(6) 618-630.

Hong, J., Bishop-Fitzpatrick, L., Smith, L. E., Greenberg, J. S., \& Mailick, M. R. (2016). Factors associated with subjective quality of life of adults with Autism Spectrum Disorder: Self-report versus maternal reports. J Autism Dev Disord, 46(4), 1368-1378.

Ireland, D., Atay C., Liddle J., Bradford D., Lee, H., Rushin, O... Vogel, A. (2016). Hello Harlie: Enabling Speech Monitoring Through Chat-Bot Conversations. Studies in Health Technology and Informatics 227: 55-60.

Ireland D., Bradford D., Farr-Wharton G., (2017) Chat Mate: Building Resilience Against Bullies Using Chat-Bots for Children on the Autism Spectrum. Digital Outreach: 
Designing Technologies for Diversity, Participation and Social Inclusion, an OZCHI workshop. Brisbane, Australia.

Ireland, D., Bradford, D., \& Silvera-Tawil, D. (2017) Research in autism-friendly technology needs to improve to make a real difference for people, The Conversation, Retrieved from https://theconversation.com/research-in-autism-friendly-technology-needs-toimprove-to-make-a-real-difference-for-people-71618.

Jordan, R. (2013). Autism with Severe Learning Difficulties: London: Souvenir Press.

Kreiser, N. L., \& White, S. W. (2014). ASD in females: are we overstating the gender difference in diagnosis? Clinical Child and Family Psychology Review, 17(1), 67-84.

Li, Q. (2007). Bullying in the new playground: Research into cyberbullying and cyber victimisation. Australasian Journal of Educational Technology, 23(4).

Lodge, J. (2014). Children who Bully at School. Australian Institute of Family Studies. CFCA Paper No. 27.

National Autism Resources (2017). The Picture Exchange Communication System (PECS). Retrieved from https://www.nationalautismresources.com/the-picture-exchangecommunication-system-pecs/

NDIS (2018). COAG Disability Reform Council June Quarterly Report. National Disability Insurance Scheme. Canberra, Australia.

Ospina, M. B., Seida, J. K., Clark, B., Karkhaneh, M., Hartling, L., Tjosvold, L., ... Smith, V. (2008). Behavioural and developmental interventions for autism spectrum disorder: a clinical systematic review. PLoS One, 3(11), e3755.

Patel, V. B., Preedy, V. R., \& Martin, C. R. (2014). Comprehensive Guide to Autism. Springer.

Sapouna, M., Wolke, D., Vannini, N., Watson, S., Woods, S., Schneider, W., \& André, E. (2010). Virtual learning intervention to reduce bullying victimization in primary school: a controlled trial. Journal of Child Psychology and Psychiatry, 51(1), 104-112.

Scott, M., Jacob, A., Hendrie, D., Parsons, R., Girdler, S., Falkmer T., \& Faulkmer, M., (2017) Employers' perception of the costs and the benefits of hiring individuals with autism spectrum disorder in open employment in Australia. PLoS ONE 12(5), e0177607.

Tissot, C., \& Evans, R. (2010) Visual Teaching Strategies for Children with Autism, Early Child Development and Care, 173(4), 425-433.

Ttofi, M. M., \& Farrington, D. P. (2011). Effectiveness of school-based programs to reduce bullying: A systematic and meta-analytic review. Journal of Experimental Criminology, $7(1), 27-56$.

Watson, A., Bickmore, T., Cange, A., Kulshreshtha, A., \& Kvedar, J. (2012). An internet-based virtual coach to promote physical activity adherence in overweight adults: randomized controlled trial. Journal of Medical Internet Research, 14(1).

Wehman, P. H., Schall, C. M., McDonough, J., Kregel, J., Brooke, V., Molinelli, A., \& Thiss, W. (2014). Competitive employment for youth with autism spectrum disorders: Early results from a randomized clinical trial. Journal of Autism and Developmental Disorders, 44(3), 487-500.

Wong, C., Odom, S. L., Hume, K. A., Cox, A. W., Fettig, A., Kucharczyk, S., Schultz, T. R. (2015). Evidence-based practices for children, youth, and young adults with autism spectrum disorder: A comprehensive review. Journal of Autism and Developmental Disorders, 45(7), 1951-1966. 